\title{
Duración de la gestación en el ganado bovino criollo costeño con cuernos
}

\author{
Duration of gestation in creole cattle breed Costeño con Cuernos
}

\author{
GONZÁLEZ T. MARCO ${ }^{*}$, M.Sc, OSSA S. GUSTAVO² Ph.D, PÉREZ G. JUAN² Ph.D.
}

${ }^{1}$ Universidad de Córdoba. Facultad de Medicina Veterinaria y Zootecnia. Departamento de Ciencias Pecuarias. Instituto de Investigaciones biológicas del Trópico IIBT. Montería, Colombia. ${ }^{2}$ Corporación Colombiana de Investigación Agropecuaria CORPOICA. Cereté, Colombia.

\section{Keywords:}

Gestation, bovine, creole cattle.

\section{Abstract}

The aim of this study was to determine the duration of pregnancy in the Creole cattle breed Costeño con Cuernos and establish whether factors such as the sex of the calf or order delivery could intervene in the duration of this period. A retrospective study was conducted in a herd of females of Center for Agricultural Research, Turipaná, Cerete, Colombia. It was considered as length of gestation, the interval between the date of effective service and date of delivery time. The period considered was between the years 1969 and 1988. The overall average length of gestation of 184 cases was $288.6 \pm 5.7$ days. Calf sex influenced $(p<0.05)$ on the length of gestation, and was longer lasting when it was male, when was female (290 $\pm 288 \pm 5.2$ vs. 6.0 ). Cows were arranged according to the number of labor into three groups: group I (one and two deliveries), group II (three and four deliveries) and group III ( 5 or more deliveries). It was found that the sex of the fetus was a cause of variation in the length of gestation of cows Costeño con Cuernos $(p<0.05)$ among the cows in groups I and II, and between group II and III.

\section{Resumen}

Determinar la duración de la gestación en la raza bovina criolla Costeño con Cuernos y establecer si factores tales como el sexo del ternero o el orden de partos podrían intervenir en la duración de este periodo. Se realizó un estudio retrospectivo en un hato de hembras del Centro de Investigación Agropecuaria, Turipaná, Cereté, Colombia. Se consideró como duración de la gestación, el intervalo de tiempo transcurrido entre la fecha del servicio efectivo y la fecha del parto. El periodo considerado fue entre los años de 1969 y 1988. El promedio general de la duración de la gestación de 184 casos fue de 288,6 \pm 5,7 días. El sexo del ternero influyó $(p<0,05)$ sobre la duración de la gestación, y resultó de mayor duración cuando fue macho, que cuando fue hembra (290 $\pm 5,2$ vs. $288 \pm 6,0$ ). Las vacas fueron ordenadas de acuerdo con el número del parto en tres grupos: grupo I (uno y dos partos), grupo II (tres y cuatro partos) y grupo III (5 o más partos). Se encontró que el sexo del feto fue una causa de variación en la duración de la gestación de las vacas Costeño con Cuernos $(p<0,05)$ entre las vacas de los grupos I y II, así como entre las vacas de los grupos II y III. 


\section{Introducción}

Las razas nativas tropicales latinoamericanas descienden de animales que llegaron durante la conquista española en 1492, y a través de más de 500 años de selección natural, han logrado un proceso de adaptación al medio que se ve reflejado en la expresión de algunos caracteres de importancia económica que pueden ser utilizados para aumentar su producción animal. En Colombia se desarrollaron siete razas de bovinos criollos, la raza Casanareño y la Sanmartinero, desarrolladas en el Piedemonte Llanero y en las sabanas del oriente Colombiano. La raza Blanco Orejinegro (BON) en la región Andina, la raza Chino Santandereano en el centro y norte de la Cordillera Oriental y la raza Hartón del Valle formada en el Valle del Cauca. En la región Caribe predominaron las razas Costeño con Cuernos y el Romosinuano (PRIMO, 1992).

La raza Costeño con cuernos (CCC) fue utilizada como productora de leche y carne, y en 1986 fueron contabilizados 476 animales puros conservados por CORPOICA en el Centro de Investigaciones Turipaná, Cereté, Colombia. El CCC es un bovino de tamaño mediano, su color varía entre bayo claro y rojo cereza, cabeza con cuernos delgados, cola de inserción alta y escasa borla. La conformación de las vacas revela aptitud para la producción de leche, con ubre glandular de apariencia colgante, medianas y con venas mamarias bien desarrolladas (PRIMO, 1992).

La información relacionada con los aspectos reproductivos como la duración de la gestación de esta raza es desconocida. Una evaluación estadística del periodo de gestación puede ser requerida en estudios experimentales con el fin de determinar si su duración ha sido afectada por algún tratamiento. Además, es necesario el conocimiento de la variación normal de la duración de la gestación en una raza determinada para planificar el manejo de los animales en los estados de preñez avanzada. Adicionalmente el conocimiento de la duración de la gestación en esta raza criolla es necesario para la caracterización de la misma. El objetivo de este estudio fue determinar la duración de la gestación en el ganado Costeño con Cuernos y establecer si factores como el sexo de la cría o el orden de partos podrían incidir en este periodo.

\section{Materiales Y Métodos}

Tipo de estudio y zona de estudio. Se realizó un estudio retrospectivo a través de los resultados obtenidos de la duración de la gestación de hembras del hato Costeño con Cuernos, perteneciente al Centro de Investigación Turipaná (CORPOICA), localizado en el municipio de Cereté, departamento de Córdoba, Colombia. La zona de estudio presenta una precipitación anual promedio de $1.100 \mathrm{~mm}$, humedad relativa del $83 \%$, altura de $13 \mathrm{msnm}$ y una temperatura promedio anual de $28^{\circ} \mathrm{C}$.

Obtención de datos y análisis de la información. Se obtuvieron datos sobre el número de partos, duración de la gestación y sexo de la cría de hembras Costeño con Cuernos que presentaron fecha se servicio (monta natural) y fecha de parto entre los años de 1969 y 1988. Se conformaron tres grupos de animales de acuerdo con el número de partos: El grupo I correspondió a vacas de uno y dos partos, el grupo II a vacas de tres y cuatro partos y el grupo III a vacas de cinco o más partos. El rango de número de partos de los animales estudiados fue entre uno y doce.

Con el propósito de determinar si el número de partos o el sexo de la cría afectaban la duración de la gestación, se realizó una prueba de "t" de Student con un nivel de significancia del $5 \%$. Los resultados obtenidos fueron analizados mediante estadística descriptiva, desviación estándar y coeficiente de variación.

\section{Resultados y Discusión}

Este es el primer estudio en Colombia en el que se informa la duración de la gestación en el ganado Costeño con Cuernos. La distribución del periodo de gestación de 184 casos de hembras bovinas analizadas se describe en la Tabla 1. El $80 \%$ de las gestaciones tuvieron una duración entre 281 y 295 días. La media de la duración de la gestación encontrada (288,6 \pm 5,7 días) es similar a la informada para las razas criollas brasileñas Caracú y Pitangueiras, reportadas por PEREIRA et al.,(1979) y LOBO et al.,(1980), respectivamente. De igual forma, los resultados 
de este estudio coinciden con la media reportada para la raza criolla venezolana Carora (VALLE, 1985) y para la raza Criolla Cubana reportada por RIZO et a.,2002.

Tabla 1. Distribución de la duración de la gestación en hembras Costeño con Cuernos.

\begin{tabular}{cc}
\hline $\begin{array}{c}\text { Rangos de duración de la } \\
\text { gestación en días }\end{array}$ & $\begin{array}{c}\text { Frecuencia de animales \% } \\
\text { (n) }\end{array}$ \\
\hline $270-275$ & $4,34(8)$ \\
$276-280$ & $4,89(9)$ \\
$281-285$ & $13,04(24)$ \\
$286-290$ & $33,69(62)$ \\
$291-295$ & $34,78(64)$ \\
$296-300$ & $9,23(17)$ \\
\hline Total de animales & $\mathbf{1 8 4}$ \\
\hline
\end{tabular}

La duración de la gestación es un carácter condicionado genéticamente, aunque puede estar modificada por factores maternos, fetales y ambientales. Entre los factores descritos que condicionan la mayor o menor duración del período gestacional en el ganado vacuno son el sexo de la cría, la edad de la vaca, la estación de parto, el número de fetos, los criterios de selección, el genotipo fetal y el peso al nacimiento (BECERRA, 2012).

En este estudio se analizaron algunos de los factores que afectan la duración de la gestación como el orden del parto y el sexo de la cría. Se encontró que el orden de partos interviene en la duración de la gestación de hembras Costeño con Cuernos, dado a que se identificaron variaciones en el periodo de gestación entre las vacas del grupo I y II y entre las vacas del grupo II y III (Tabla 2). Se hallaron diferencias significativas entre hembras con uno y dos partos (grupo I) y hembras con dos y tres partos $(P<0,05)$, clasificando a las hembras del grupo I como las que presentaron un período de gestación menor. Estos resultados posiblemente se deben a que las vacas con uno y dos partos no se han desarrollado completamente y el peso del ternero hace que estas presenten el parto más acelerado que las pluríparas. De acuerdo con un estudio realizado por CABALLERO Y GONZÁLEZ, (1997) en la raza bovina de Lidia, reportan que vacas jóvenes con uno y dos partos
Tabla 2. Distribución de duración de la gestación de acuerdo con el número de partos.

\begin{tabular}{ccccc}
\hline Categorías & $\begin{array}{c}\text { Número de } \\
\text { observaciones }\end{array}$ & Media & D.S & C.V.*\% \\
\hline Grupo I & 38 & 288,52 & 7,09 & 2,45 \\
Grupo II & 60 & 287,30 & 6,28 & 2,18 \\
Grupo III & 86 & 289,80 & 4,16 & 1,43 \\
\hline
\end{tabular}

* Desvío estándar; ** Coeficiente de variación

presentan periodos más cortos de gestación, y que este período aumenta gradualmente con el número de partos. Asimismo, RIZO et al., 2002 reportaron que en el ganado Criollo Cubano, el número de partos influye significativamente en la duración de la gestación ( $p>0,001)$. Por otra parte, LOBO et al, (1980) reportaron pequeñas variaciones en la duración de la gestación de acuerdo con el orden de parto en la raza criolla brasileña Pitangueiras, en las cuales ocurren gestaciones más prolongadas entre el sexto y octavo parto. No obstante, este autor indicó que los coeficientes de variación fueron prácticamente idénticos, considerando que la variabilidad de este carácter es de poca importancia práctica.

En este estudio también se encontró que el sexo del feto es una causa de variación en la duración de la gestación de las vacas Costeño con Cuernos. El período de gestación de fetos macho $(289,84$ $\pm 5,24$ días) fue mayor que el de fetos hembra $(287,83 \pm 5,96)(p<0,05)$. De forma similar, estos resultados también son descritos por BECERRA et al, (2012) en bovinos de la raza Rubia Gallega. Igualmente, CABALLERO Y GONZÁLEZ, (1997) reportaron una mayor duración de la gestación de machos que de hembras, aunque no encontró diferencias significativas entre estas variables.

Las diferencias en la duración de la gestación de acuerdo con el sexo de la cría ha sido reportada con frecuencia en la literatura y esta podría ser atribuida a que los machos son más pesados que las hembras no solo al nacer, si no en todas las edades. Esto podría explicarse porque los machos presentan mayores índices de crecimiento pre y post-natal, posiblemente debido a la acción de la testosterona, la cual determina una mayor tasa metabólica (BECERRA et al, (2012); CABALLERO Y GONZÁLEZ, (1997); NA et al (1989)). Por lo tanto una de las causas del parto es el tamaño del feto, incidiendo esto en la duración de la gestación. 


\section{Conclusiones}

Este es el primer estudio en Colombia en donde se informa la duración de la gestación de bovinos de la raza criolla Costeño con Cuernos. Se encontró que este periodo presenta una media de 288,6 \pm 5,7 días, y factores como el orden de partos y el sexo de la cría tienen influencia sobre la duración de la gestación.
Agradecimientos. A todos los trabajadores de campo y técnicos de CORPOICA., Cereté, Colombia. A la Universidad de Córdoba y a la Facultad de Medicina Veterinaria y Zootecnia. Montería Colombia.

\section{Referencias}

BECERRA, J; QUINTELA, L; PEÑA, A; RUIBAL, S; DEIROS, J; BARRIO, M; DIAZ, C; GRACIA, S; HERRADÓN, P. Duración del período gestacional en la raza Rubia Gallega. Recursos Rurais. 1(2):3539. 2012

CABALLERO DE LA CALLE, J \& GONZÁLEZ, M. Influencia de diversos factores sobre la duración de la gestación en vacas bravas." Archivos de zootecnia 46(173): 81-84. 1997.

LOBO, R; VALLE, A; DUARTE, F; y WILCOX, C. Estudio fenotipico y genetico de caracteristicas reproductivas y productivas en la raza Pitangueiras. I. Duracion de la gestacion. Agronomía Tropical 30(1-6): 315-324. 1980.

NA, K.J.; HONG, S.G.; KIM, H.S. \& LEE, K.S. Environmental effects on the reproductive traits of Korean Native cattle and Charolais crossbreds on farm feeding. The Research Reports of the Rural Development Administration 31, 2: 1-6. 1989.

PRIMO, A. El ganado bovino ibérico en las Américas: 500 años después. Archivos de zootecnia. 41(154): 421-432. 1992.

RIZO, A; GUEVARA G; PAZ SARMIENTO, A; VALDÉS, J; AVILÉS, R; GÓMEZ, M Y VEGA, C. Comportamiento reproductivo del ganado vacuno Criollo Cubano. Rev. prod. anim. 14 (1):55-58. 2002

VALLE, A. 1985. Comportamiento reproductivo de vacas mestizas en cinco fincas de la región de Carora, Estado Lara. Venezuela. Zoot Trop 3(1). 